Proceedings of the XXIII Conference on Applied Crystallography, Krynica Zdrój, Poland, September 20-24, 2015

\title{
Nanoscale Deformation of GaAs Affected by Silicon Doping
}

\author{
A. MAjtykA ${ }^{a}$, R. NOWAK ${ }^{a}$ AND D. ChrobAK ${ }^{b, *}$ \\ ${ }^{a}$ Nordic Hysitron Laboratory, Department of Materials Science and Engineering, School of Chemical Technology, \\ Aalto University, 00076 Aalto, Finland \\ ${ }^{b}$ Institute of Materials Science, Faculty of Computer Science and Materials Science, University of Silesia, \\ 75 Pułku Piechoty 1A, 41-500 Chorzów, Poland
}

\begin{abstract}
Effect of silicon doping on the elastic-plastic transition of GaAs crystal is demonstrated by results of nanoindentations and $a b$ initio simulations. The performed experiments show that an increase of silicon concentration causes a decrease of the contact pressure at the onset of permanent nanodeformation of GaAs crystal. Ab initio calculations demonstrate that presence of Si atoms in the crystal lattice suppresses the shear modulus as well as the pressure of equilibrium between zinc-blende and rock-salt phases of GaAs. Furthermore, it is argued that the effect of dislocations pinning to $\mathrm{Si}$ dopants is essential for clarification of GaAs yielding.
\end{abstract}

DOI: 10.12693/APhysPolA.130.1127

PACS/topics: $64.70 . \mathrm{kg}, 61.72 . \mathrm{J}-$

\section{Introduction}

The term "elastic-plastic transition" refers to the phenomenon that breaks a reversible path of the elastic deformation, switching a material into the plastic state. When induced by nanoindentation, this critical behavior of a crystal lattice may manifest itself by the popin, i.e., specific singularity of a load-displacement curve. Interestingly, the nanoindentation experiment preformed with sharp diamond indenter serves unique way to investigate mechanical properties of dislocation-free crystals. Hence, the occurrence of pop-in is often explained by a homogeneous nucleation of dislocations that was proved not only for metals [1], but also for GaN [2] and SiC [3] semiconductors.

Interestingly, the case of semiconductor crystals is complex as understanding of their nanoscale plasticity requires consideration the phase transformations in addition to the homogeneous nucleation of linear defects. Silicon provides an example that course of deformation depends on several transformations to a new phases: Si-II and bct-5 during loading as well as Si-III, Si-XII and $\alpha$ Si during unloading [4]. Similarly, the nanoindentationinduced plasticity of GaAs is affected by the phase transformation from GaAs-I (zinc-blende (ZB), space group F-43m, semiconducting) to GaAs-II (rock-salt(RS)-like, space group $\mathrm{Cmcm}$, metallic) structure. What is more, the $\mathrm{ZB} \rightarrow \mathrm{RS}$ transformation initiates the transition from elastic to plastic state of GaAs crystal [5, 6].

Another important factor affecting structural and mechanical properties of GaAs is an atomic doping. In general, silicon as the $n$-type dopant is known to alter a wide range of GaAs optoelectronic features [7] however, impact of silicon on a course of the nanoscale elasticplastic transition is less explored. For that reason, this

*corresponding author; e-mail: dariusz.chrobak@us.edu.pl study is intended to show how silicon admixture influences the onset of the nanoindentation-induced plastic deformation of GaAs crystal. This goal is directly related to the question how silicon impurities alter both the elastic constants of ZB lattice and the equilibrium between GaAs-I and GaAs-II phases. Finally, it will be shown that the increase of silicon concentration favors the phase transformation as an origin of GaAs plastic nanodeformation and simultaneously increases the shear stress required to release dislocation activity.

\section{Results and discussion}

Nanoindentations (Hysitron TI-950 triboindenter) were performed on $1.5 \mu \mathrm{m}$ thick crystals of (001) ZBGaAs doped with silicon concentration of $1 \times 10^{+16}$, $1 \times 10^{+17}$, and $1 \times 10^{+18} 1 / \mathrm{cm}^{3}$. Thin films were grown by molecular beam epitaxy on $350 \mathrm{~mm}$ thick (100) epi-ready GaAs substrates. Figure 1 shows the results of experiments (49 for each sample) conducted with the Berkovich diamond indenter. It is clear that the increase of the silicon concentration slightly decreases the contact pressure $\left(p_{c}=F / A, F\right.$ - force, $A$ - contact area) at the elastic-plastic transition (pop-in event) of doped GaAs. The estimated averaged pop-in pressures take the values of $p_{c}\left(1 \times 10^{+16}\right) \approx 13.7 \pm 0.4, p_{c}\left(1 \times 10^{+17}\right) \approx 13.6 \pm 0.3$, and $p_{c}\left(1 \times 10^{+18}\right) \approx 13.3 \pm 0.3 \mathrm{GPa}$.

It is known from previous studies that silicon doping decreases the density of dislocations during the growth of a GaAs monocrystals [8] as well as inhibits the mobility of dislocations under applied external stress [9]. Consequently, assuming dislocation origin of GaAs plasticity, one could expect to observe an increase of the mean contact pressure of the elastic-plastic transition caused by increasing silicon concentration. However, it is not the case. The experimental data presented in Fig. 1 show the opposite effect that suggests the non-dislocation origin of the pop-in events. Indeed, the results of presented experiments are in line with the outcomes of earlier nanoelectrical [6] and loading rate dependent [10] measurements that 

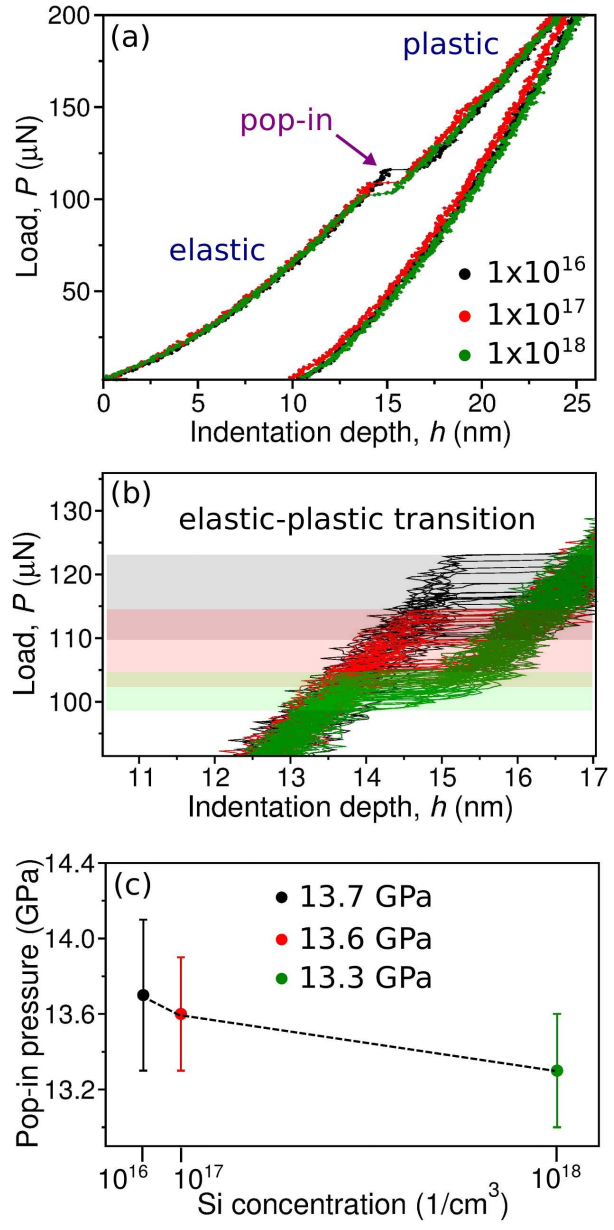

Fig. 1. Nanoindentation experiments performed for silicon doped GaAs crystal. (a) The representative $P-h$ curves corresponding to considered $\mathrm{Si}$ concentrations. (b) The details of all experimental $P-h$ curves recorded in the vicinity of the elastic-plastic transition. The systematic suppression of the load at the onset of permanent nanodeformation is clearly demonstrated. (c) The relationship between mean value of the pop-in contact pressure and Si concentration.

demonstrated GaAs-I $\rightarrow$ GaAs-II phase transformation as the origin of nanoindentation induced plastic deformation of GaAs. However, in order to confirm the phase transformation as the cause of the elastic-plastic transition in Si doped GaAs one ought to prove that the presence of silicon dopants reduces the pressure of GaAs-I $\rightarrow \mathrm{GaAs}-\mathrm{II}$ phase transformation and simultaneously increases the critical shear stress required to initiate the dislocation processes. Realization of these particular tasks makes it necessary to estimate the silicon impact on both the elastic constants of GaAs-I and the equilibrium between GaAs-I and GaAs-II phases.

The $a b$ initio calculations were carried out using the Quantum-Espresso code [11]. The norm-conserving pseudopotentials for $\mathrm{Ga}$, As, and $\mathrm{Si}$ were adapted from the PSlibrary [11] while the other basic details of calculations can be summarized as follows: the exchange-correlation energy functional was expressed by the Perdew-Zunger approximation [12], the $k$-point space was discretized with $7 \times 7 \times 7$ Monkhorst-Pack mesh [13], the plane-wave basis set had the kinetic energy cutoff of 60 Ry.

In order to model the crystal lattice with various silicon concentration two supercells (each of them composed of $2 \times 2 \times 2$ cubic elementary cells) were determined for both the ZB and the RS structures of GaAs. The real highpressure GaAs-II phase was replaced by the rock-salt one because of the tiny difference between their ground state energies [14]. Simulation of silicon doping was realized by the substitutional $\mathrm{Si}_{\mathrm{Ga}}$ point defects for which the gallium atoms were simply replaced by silicon ones. This choice was motivated by the fact that the formation energy of $\mathrm{Si}_{\mathrm{Ga}}$ is lower than the formation energy of $\mathrm{Si}_{\mathrm{As}}$ point defect. In that way, two different silicon concentration $n_{1}, n_{2}\left(n_{1}<n_{2}\right)$ were defined by placing Si atom in the $(0.5,0.5,0.5)_{\mathrm{Ga}}$ and two $\mathrm{Si}$ atoms in $(0,0,0)_{\mathrm{Ga}}$, $(0.5,0.5,0.5)_{\mathrm{Ga}}$ positions, respectively. The elastic constants of ZB lattice and equilibrium conditions for $\mathrm{ZB}$ and RS lattices were also simulated for undoped GaAs. The coordinates of the first and the second neighbors of the silicon atoms were relaxed until the atomic forces decreased below the threshold of $10^{-4}$ Ry/a.u. ${ }^{3}$.

\section{TABLE I}

Results of $a b$ initio calculations of lattice constant $a[\AA]$, elastic constants $c_{i j}[\mathrm{GPa}]$, shear moduli $G$ [GPa], Poisson ratio $\nu$, and critical shear stress for homogeneous nucleation of dislocation $\tau_{c}$ [GPa].

\begin{tabular}{c|c|c|c}
\hline \hline & undoped & $n_{1}$ & $n_{2}$ \\
\hline$a$ & 5.613 & 5.619 & 5.622 \\
$c_{11}$ & 116.68 & 108.38 & 98.18 \\
$c_{12}$ & 52.66 & 52.01 & 51.11 \\
$c_{44}$ & 59.19 & 47.88 & 36.82 \\
$G$ & 48.32 & 40.00 & 31.50 \\
$\nu$ & 0.23 & 0.26 & 0.30 \\
$\tau_{c}$ & 5.22 & 4.42 & 3.58
\end{tabular}

The cubic lattice parameter $a$ corresponding to a minimum of the ground state energy $E$ and the elastic constants $c_{i j}$ were calculated for zinc-blende GaAs by application of volumetric, tetragonal, and rhombohedral lattice distortions. The results of ab initio calculations (refer to Fig. 2 and Table I) show that the lattice constant of the ZB-GaAs slightly increases taking the values of $5.613,5.619$, and $5.622 \AA$ for substitutional $\mathrm{Si}_{\mathrm{Ga}}$ point defect concentrations of $n=0, n_{1}=7.1 \times 10^{+20}$ and $n_{2}=1.4 \times 10^{+21} 1 / \mathrm{cm}^{3}$, respectively. Simultaneously, the values of all calculated elastic moduli decrease. In order to estimate the critical shear stress $\tau_{c}$ for homogeneous dislocation's nucleation we used equation, applicable for dislocations in form of circular loop: $\tau_{c}=3 G /\left(\pi e^{3}\right)$ $(2-\nu) /(1-\nu)$, where $G$ and $\nu$ mean shear modulus and Poisson ratio, respectively. The calculated $\tau_{c}$ decreases with an increase of the silicon concentration (Table I).

The effect of $\mathrm{Si}_{\mathrm{Ga}}$ point defects on equilibrium of $\mathrm{ZB}$ and RS phases of GaAs was analyzed on the basis of 

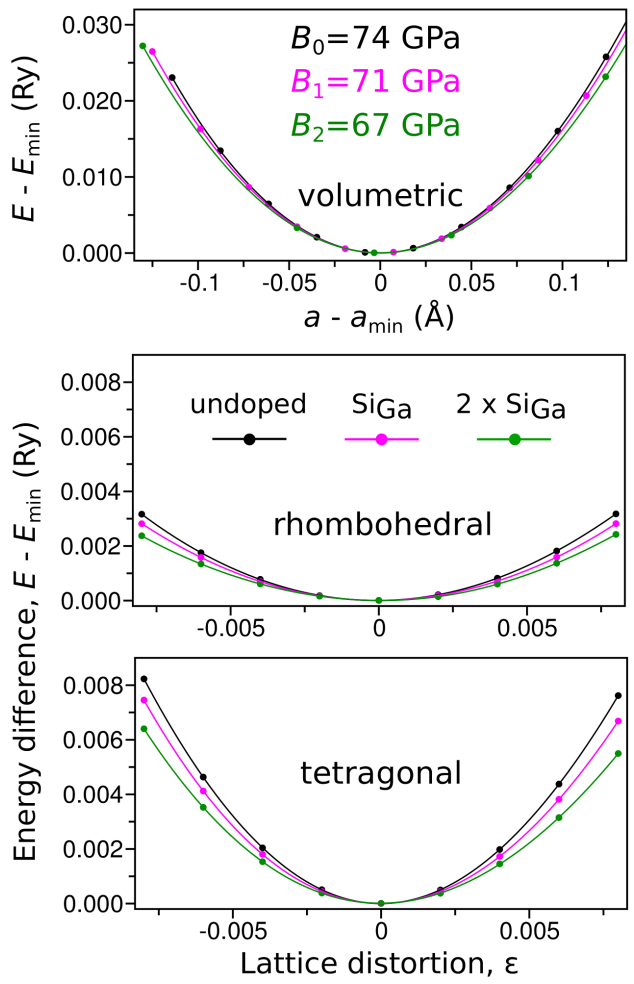

Fig. 2. Ab initio simulations performed for undoped and silicon doped zinc-blende GaAs structure showing an effect of volumetric, rhombohedral, and tetragonal lattice distortions on the ground state energy. The increase of silicon concentration results in "softening" of the zinc-blende lattice.

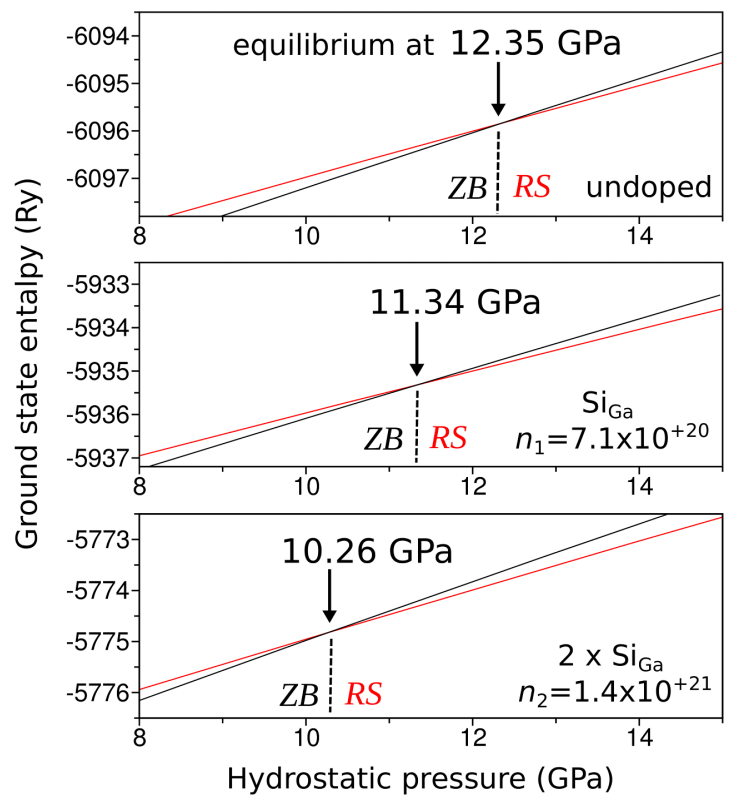

Fig. 3. Effect of $\mathrm{Si}_{\mathrm{Ga}}$ point defects concentration on the ground state enthalpy of zinc-blende and rock-salt structures of GaAs whose common value for particular value of the hydrostatic pressure defines the phase equilibrium. The calculated equilibrium pressure is suppressed by an increase of silicon doping.
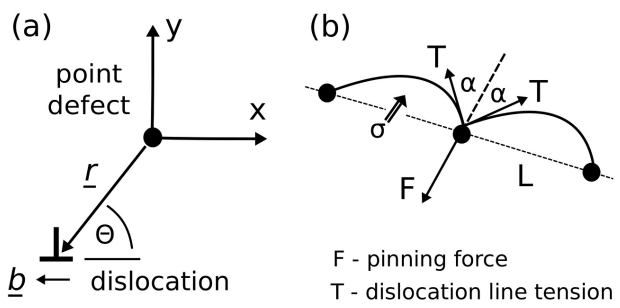

Fig. 4. The interaction between point-defects and dislocations is considered within the classical theory of elasticity. (a) The point defect is located in the origin of the coordinate system while the dislocation's line crosses the $x-y$ plane at the point indicated by vector $r$. (b) Schematic of pinning phenomenon. An external shear stress $\sigma$ results in force $f=\sigma b$ acting on the unit length of the dislocation that bows out until the force $f L$ is in equilibrium with the line tension $T$ : $\sigma b L=2 T \cos \alpha$.

$E_{\mathrm{ZB}}(V), E_{\mathrm{RS}}(V)$ relationships that describe variation of the ground state energy with respect to the supercell volume. The hydrostatic pressure at the phase equilibrium $p_{\text {eq }}$ was estimated from the crossing point of $H_{\mathrm{ZB}}(V)$ and $H_{\mathrm{RS}}(V)$ graphs, where $H=E+p V$ means the ground state enthalpy. As shown in Fig. 3, the pressure $p_{\text {eq }}$ decreases taking the values of $12.35,11.34,10.26 \mathrm{GPa}$ for increasing silicon concentration. The calculated values are consistent with the results of other studies [15].

The nanoindentation experiments (Fig. 1) have shown that the contact pressure at the onset of GaAs plastic deformation decreases with an increase of Si doping. Unexpectedly, the $a b$ initio simulations suggested that this effect can be driven by the phase transformation or the homogeneous nucleation of dislocations as both the pressure of $\mathrm{ZB} \rightarrow \mathrm{RS}$ transformation and the critical stress for homogeneous dislocation's nucleation decrease with increasing silicon concentration. In view of these findings, one cannot indicate the unique cause of the nanoindentation induced plastic deformation of GaAs doped by silicon. However, the dislocation activity includes not only homogeneous dislocation nucleation but also the attractive interaction of dislocations with the point defects.

The energy of elastic interaction between the edge dislocation and the point defect is given by [16]: $E_{\text {int }}=$ $-\beta G b \sin (\theta) / r$, where $G$ is the shear modulus, $b$ refers to a magnitude of the Burgers vector, $r$ and $\theta$ are cylindrical coordinates of dislocation line as depicted in Fig. 4. The $\beta$ parameter takes positive value when an introduction of the point defect causes an increase of the lattice constant, which is, in fact (Table I), the case of $\mathrm{Si}_{\mathrm{Ga}}$ impurity. The attractive force $F$ acting on dislocation is then characterized by $F_{\theta}=-\beta G b \cos (\theta) / r$ and $F_{r}=-\beta G b \sin (\theta) / r^{2}$ components and takes the maximal magnitude $F=\beta G / b$ for $\theta=\pi / 2$ and in the vicinity of dislocation's core i.e., for $r=b$. This is the force that should be defeated by the external shear stress in order to release dislocation line from the pinning $\mathrm{Si}$ atom.

Let us now assume $L$ to be the average distance between dopants (Fig. 4). In order to break away the dislocation from the impurity one should apply the shear 
stress $\sigma^{c}$ that satisfies the following equation: $\sigma^{c} b L=$ $\beta G / b$. This shows that $\sigma^{c}$ should be proportional to the shear modulus and inversely proportional to the average distance between point defects. Thus, neglecting variation of $b$ and $\beta$ parameters with respect to dopant concentration, the stress ratio $\sigma_{1}^{c} / \sigma_{2}^{c}=G_{1} L_{2} /\left(G_{2} L_{1}\right)$, where the lower indices correspond to $n_{1}=7.1 \times 10^{+20}$ and $n_{2}=1.4 \times 10^{+21}$ doping levels. Using the Voigt approximation of the shear modulus $G_{v}$ and noting that $L_{1}=2 L_{2}$ one can write $\sigma_{1}^{c}=0.5 \sigma_{2}^{c}$ i.e., the shear stress required to move the edge dislocation from the pinning atoms becomes greater for higher silicon concentration. In contrast to just established behavior of $\sigma^{c}$, the pressure $p_{\text {eq }}$ at the onset of the $\mathrm{ZB} \rightarrow \mathrm{RS}$ transformation decreases (Fig. 3). Consequently, the result of nanoindentation experiments (Fig. 1) can be explained in terms of the phase transformation from semiconducting to metallic structure of GaAs.

\section{Conclusion}

In summary, this letter confirms stimulated by silicon decrease of contact stress at the onset of nanoindentation induced plastic deformation of GaAs crystal. The computational analysis of silicon impact on dislocation activity along with simulation of $\mathrm{ZB}$ and RS phase equilibrium proved non-dislocation character of the elasticplastic transition in $\mathrm{Si}$ doped GaAs crystals.

\section{Acknowledgments}

This work was supported by National Science Center of Poland under grant No. DEC-2012/05/B/ST8/02945. The author gratefully acknowledges CSC - IT Center for Science Ltd for the allocation of computational resources.

\section{References}

[1] J. Li, K.J. Van Vliet, T. Zhu, S. Yip, S. Suresh, Nature 418, 307 (2002).
[2] M. Fujikane, T. Yokogawa, S. Nagao, R. Nowak, Appl. Phys. Lett. 101, 201901 (2012).

[3] I. Szlufarska, A. Nakano, P. Vashishta, Science 309, 911 (2005).

[4] J.E. Bradby, J.S. Williams, M.V. Swain, Phys. Rev. B 67, 085205 (2003).

[5] D. Chrobak, K. Nordlund, R. Nowak, Phys. Rev. Lett. 98, 045502 (2007).

[6] R. Nowak, D. Chrobak, S. Nagao, D. Vodnick, M. Berg, A. Tukiainen, M. Pessa, Nature Nanotechnol. 4, 287 (2009).

[7] J.S. Blakemore, J. Appl. Phys. 53, R123 (1982).

[8] E.D. Bourret, M.G. Tabache, A.G. Elliot, Appl. Phys. Lett. 50, 1373 (1987).

[9] I. Yonenaga, K. Sumino, J. Appl. Phys. 62, 1212 (1987).

[10] D. Chrobak, K.-H. Kim, K.J. Kurzydlowski, R. Nowak, Appl. Phys. Lett. 103, 072101 (2013).

[11] P. Giannozzi, S. Baroni, N. Bonini, M. Calandra, R. Car, C. Cavazzoni, D. Ceresoli, G.L. Chiarotti, M. Cococcioni, I. Dabo, A. Dal Corso, S. de Gironcoli, S. Fabris, G. Fratesi, R. Gebauer, U. Gerstmann, C. Gougoussis, A. Kokalj, M. Lazzeri, L. Martin-Samos, N. Marzari, F. Mauri, R. Mazzarello, S. Paolini, A. Pasquarello, L. Paulatto, C. Sbraccia, S. Scandolo, G. Sclauzero, A.P. Seitsonen, A. Smogunov, P. Umari, R.M. Wentzcovitch, J. Phys. Condens. Matter 21, 395502 (2009).

[12] J.P. Perdew, A. Zunger, Phys. Rev. B 23, 5048 (1981).

[13] H.J. Monkhorst, J.D. Pack, Phys. Rev. B 13, 5188 (1976).

[14] S.B. Zhang, M.L. Cohen, Phys. Rev. B 39, 1450 (1989).

[15] A. Mujica, A. Rubio, A. Munoz, R.J. Needs, Rev. Mod. Phys. 75, 863 (2003).

[16] J.P. Hirth, J. Lothe, Theory of Dislocations, McGraw-Hill, New York 1968. 